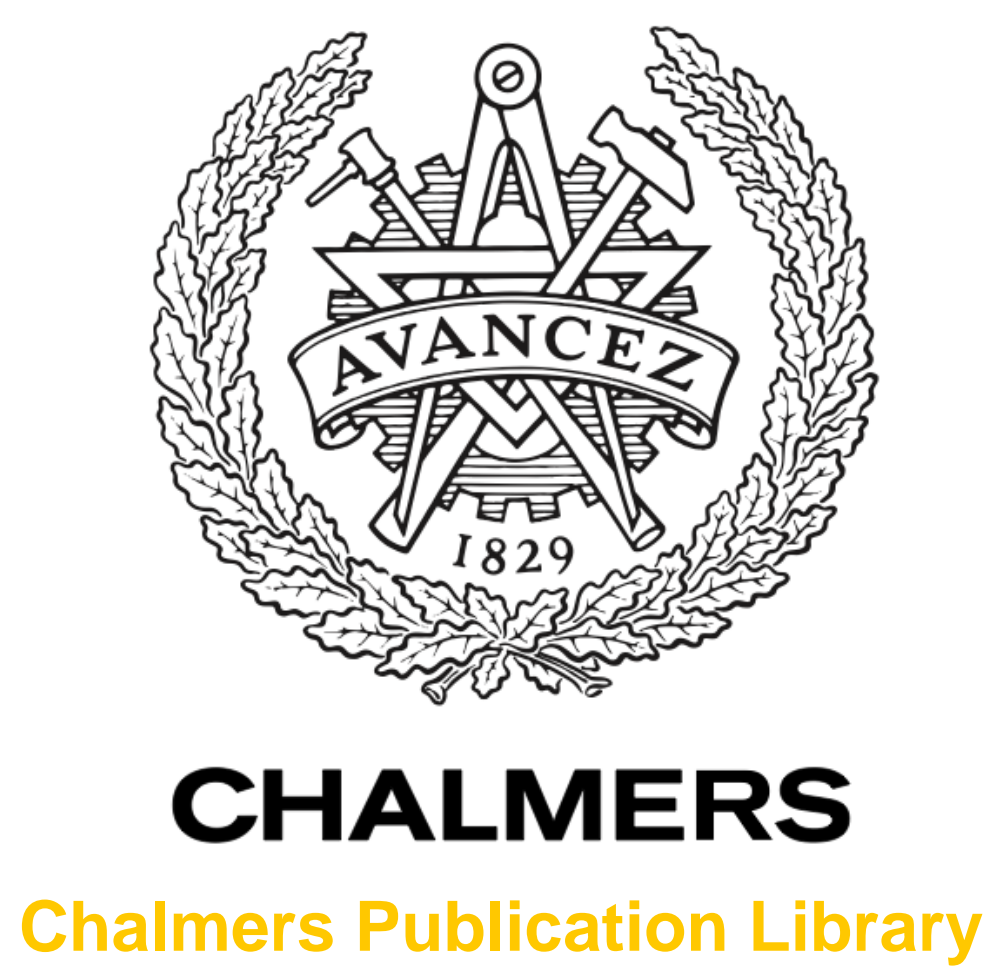

\title{
Optimum detection in presence of nonlinear distortions with memory
}

This document has been downloaded from Chalmers Publication Library (CPL). It is the author's version of a work that was accepted for publication in:

Proc.l European Conference on Optical Communication (ECOC)

Citation for the published paper:

Liga, G. ; Alvarado, A. ; Agrell, E. et al. (2015) "Optimum detection in presence of nonlinear distortions with memory". Proc. $\backslash$ European Conference on Optical Communication (ECOC)

Downloaded from: http://publications.lib.chalmers.se/publication/231109

Notice: Changes introduced as a result of publishing processes such as copy-editing and formatting may not be reflected in this document. For a definitive version of this work, please refer to the published source. Please note that access to the published version might require a subscription. 


\title{
Optimum Detection in Presence of Nonlinear Distortions with Memory
}

\author{
Gabriele Liga $^{(1)}$, Alex Alvarado(1), Erik Agrell(2)${ }^{(2)}$ Marco Secondini( ${ }^{(3)}$, Robert I. Killey(1) and Polina Bayvel ${ }^{(1)}$ \\ (1) Optical Networks Group, University College London, EE Dept., London, UK, g.liga@ee.ucl.ac.uk \\ (2) Department of Signals and Systems, Chalmers University of Technology, Gothenburg, Sweden \\ (3) TeCIP Institute, Scuola Superiore Sant'Anna, Pisa, Italy
}

\begin{abstract}
The performance of nonlinearity-tailored detection for single channel, single span optical fibre systems is studied. Monotonically decreasing bit error rate with transmitted power can be achieved without any nonlinearity compensation.
\end{abstract}

\section{Introduction}

The need to explore the fundamental limits of single mode optical fibre transmission, driven by the ever-growing capacity demand, has recently revived interest in regenerative systems ${ }^{1}$, which represent the upper bound on capacity of multispan repeatered systems. Despite the single span fibre channel being a much simpler case than the multispan one, the structure of the optimum receiver remains to be identified.

In multispan systems with coherent detection common receiver-side digital signal processing techniques, such as digital backpropagation (DBP), aim to improve the performance of optical fibre systems by compensating for fibre nonlinearities. However DBP has recently been shown to be outperformed by improved detection techniques that account for nonlinearity and memory of the channel ${ }^{2,3}$. Given a certain noisy observation from the channel, these strategies aim to minimise the error probability based on the noise distribution. For the multispan case, this distribution is unavailable, and thus, suboptimal approximated solutions based on statistical models ${ }^{2}$ or factor graphs ${ }^{3}$ have been proposed. Additionally, unlike the single span case, the interaction between signal and amplified spontaneous emission (ASE) noise ${ }^{4}$ limits the signal-to-noise ratio (SNR) at the receiver and, as a result, the uncoded bit-error rate (BER) has a lower bound even with improved detection.

In this work the problem of optimum detection in the single span fibre channel is studied, to the best of our knowledge, for the first time. A single span of fibre followed by an amplifier represents the building block of a multi-span system, and thus, optimising its performance sets higher upper bounds on the performance of any optical fibre transmission system. In addition, the problem of optimal detection of signals transmitted through a nonlinear channel with memory is theoretically important since it represents a preliminary step in the design of optimal receivers for more complex channel models.

This work shows the performance of a nearoptimum detector accounting for nonlinear distortions and memory in the unrepeatered fibre system. Despite the significant nonlinearity at the high values of transmitted powers used, the uncoded BER can be made arbitrarily low.

\section{System Model}

A typical EDFA-amplified single span fibre system, as shown in Fig.1, is analysed in this work. The transmitted signal is $x(t)=\sqrt{P} \sum_{k=0}^{+\infty} x_{k} p(t-$ $k T$ ), where $x_{k}$ are normalised equally likely complex symbols transmitted at rate $R_{s}=\frac{1}{T}, p(t)$ is the modulation pulse normalised to have unitary energy, and $P$ is the transmitted power.

The signal propagation is described by the nonlinear Schrödinger equation (NLSE), and the ASE noise added by the preamplifier is modeled as an additive white Gaussian noise (AWGN) process $n(t)$. The preamplifier is assumed to ideally compensate for the span loss and to have an ideal noise figure of $3 \mathrm{~dB}$. The SNR at the preamplifier output is given by $\mathrm{SNR}=\frac{P \exp (-\alpha L)}{h \nu B}$ where $P$ is the transmitted power, $B$ is the reference bandwidth over which the SNR is measured (typically corresponding to the receiver bandwidth), $\alpha$ is the attenuation constant, $L$ is the fibre span length and $h \nu$ is the photon energy. In particular, if $B=R_{s}$, the above SNR corresponds to the one at the output of a matched filter (MF).

Chromatic dispersion compensation (CDC) for a single span of fibre is performed after the EDFA amplifier, either by using dispersion compensating fibre or by performing electronic CDC. The memory of the channel, due to the dispersive ef- 


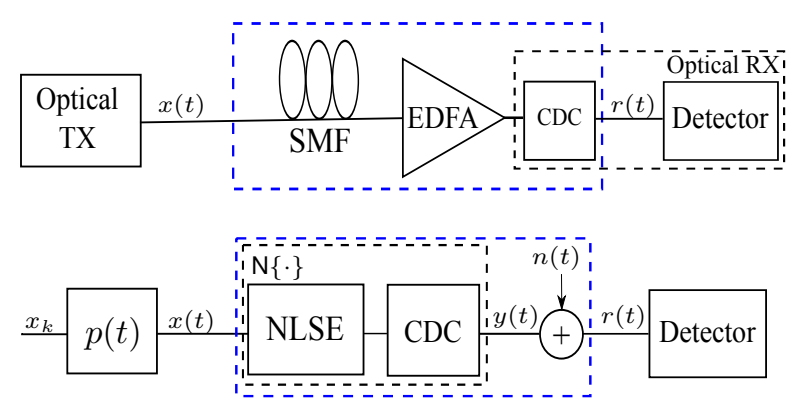

Fig. 1: Single span system and equivalent channel schematic.

fects, is therefore significantly reduced, although not entirely cancelled because of the nonlinearitydispersion interaction. This allows to simplify the structure of the subsequent optimum detector.

As shown in Fig. 1 it is assumed that the channel, from the detector standpoint, can be equivalently regarded as a nonlinear operator $\mathrm{N}\{\cdot\}$ formed by the cascade of the NLSE operator and the CDC block. The signal $r(t)$ observed at the detector can be therefore written as $r(t)=y(t)+$ $n(t)$, where $y(t)=\mathrm{N}\{x(t)\}$.

\section{Optimum Detection}

The problem of optimum detection consists of two key stages: i) extracting a set of random variables from the observed signal called a "sufficient statistic"; ii) detecting the transmitted signal according to some specific criterion, which in the case of minimising the probability of wrong detection is the maximum a posteriori probability (MAP) criterion. In the typical case of equally likely transmitted signals, the maximum likelihood (ML) strategy is equivalent to the MAP decision. A sufficient statistic $^{5}$ is a set of variables for which the observation of any other additional variable would not change the a posteriori probability, hence not resulting in any improvement in the performance of the decision strategy.

In a channel with memory, in order to have a sufficient statistic, the observation needs to be extended to multiple symbol periods. If $\mathbf{X}$ is a sequence of $K$ transmitted symbols $\mathbf{X}=$ $\left[x_{0}, x_{1}, \ldots, x_{K-1}\right]$ and $\mathbf{R}=\left[r_{0}, r_{1}, \ldots, r_{K N_{s}-1}\right]$ is a vector of observations ( $N_{s}$ per symbol), extracted from the received signal $r(t)$, making decisions based on the likelihood $p(\mathbf{R} \mid \mathbf{X})$ is referred to as maximum likelihood sequence detection (MLSD).

The operator $\mathrm{N}\{\cdot\}$ maps the transmitted "sequence-wise" signal space into a different one. Therefore, when a MF is used at the receiver, the collection of samples $\mathbf{R}$ are not in general a sufficient statistic for $\mathbf{X}$ (just a subset of it) and as a result the detection process is suboptimal.

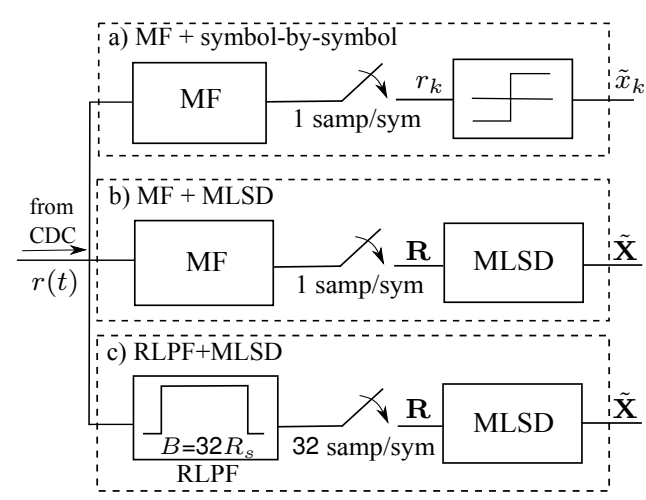

Fig. 2: Detection stategies considered in this work.

\section{Implementation and Numerical Results}

The method used in this work to extract the sufficient statistic consists of passing the received signal through a rectangular low-pass filter (RLPF) and sampling the output at $N_{s}$ samp/symbol, similar to the approach proposed in ${ }^{6}$. As also discussed in ${ }^{6}$, if the RLPF bandwidth is sufficiently large to entirely contain the received signal bandwidth (and $N_{s}$ is adjusted accordingly) the Nyquist sampling theorem guarantees that $\mathbf{R}$ represents a sufficient statistic for the sequence of symbols $\mathrm{X}$ and therefore the $\mathrm{ML}$ decision is optimal.

The simulated system comprises a single polarisation, 32 GBaud, non-return-to-zero QPSK channel transmission. At the receiver ideal CDC is applied followed by the detection stage. A comparison between the three different detection schemes shown in Fig. 2 was then carried out. In the first case (Fig. 2a) the typical MF and symbol-by-symbol detector was implemented. In the second case (Fig. 2b) the MLSD detection was performed using the output of a MF sampled at 1 samp/symbol. The last detection strategy (Fig. 2C) consists in an RLPF with bandwidth $B=32 R_{s}$, subsequently sampled at 32 sam$\mathrm{p} / \mathrm{symbol}$. This guarantees that the received signal bandwidth is correctly represented even with a significant bandwidth expansion. The MLSD is implemented using the Viterbi algorithm (VA) whose state size was varied between $3\left(4^{3}=64\right.$ states) and 7 symbols ( $4^{7}=16386$ states) to match the channel memory.

In Fig. 3 the BER vs. power $P$ (and SNR) is shown for $L=350 \mathrm{~km}$. The RLPF+MLSD achieves significant gains compared to the MF+symbol-bysymbol detection case. If the memory of the detector is limited ( 3 or 5 symbols), the BER reaches a minimum, however, for the range of powers shown, a 7-symbol VA is sufficient to ensure that the BER monotonically decreases with power. This indicates that the channel memory 


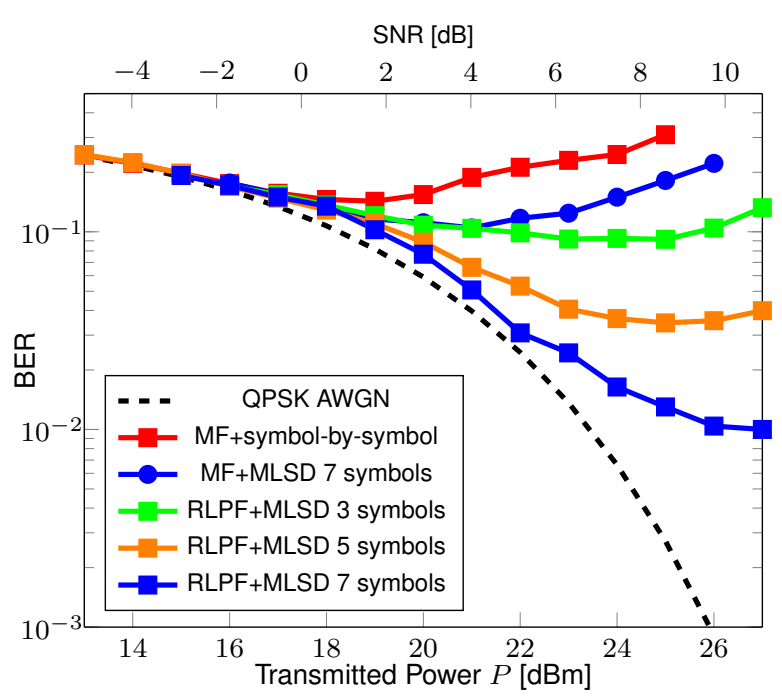

Fig. 3: Uncoded BER vs. $P$ at $L=350 \mathrm{~km}$ for different detection strategies.

increases as $P$ is increased (as confirmed by preliminary simulative results not shown here). The suboptimality of using the detection strategy in Fig. $2 b$ is shown by the green curve with circle markers. The penalty indicates that despite detection being carried out by observing samples over several symbol periods, the signal space obtained by using a matched filter at each symbol period is significantly reduced compared to the actual received one.

To quantify how the uncoded BER performance improvement translates to achievable transmission rates, the mutual information (MI) of an equivalent binary symmetric channel (BSC) was calculated. Such channel is obtained by performing hard decision on the transmitted sequences and subsequent symbol demapping. The MI was obtained as $\mathrm{MI}=1+p \log _{2}(p)+(1-p) \log _{2}(1-p)$, where $p$ is the minimum BER in the range of transmitted powers shown in Fig. 3. The resultant transmission rates vs. span length are shown in Fig. 4 for the different detection strategies analysed in this paper. For the MLSD strategies only the best case (7 symbols) was considered.

The results in Fig. 4 show that implementing a RLPF+MLSD detector up to 7 symbols and $P \leq$ $27 \mathrm{dBm}$ provides an increase in span length of $\approx 50 \mathrm{~km}$ for most of the transmission rates compared to the MF+symbol-by-symbol detection and of $\approx 40 \mathrm{~km}$ compared to the MF+MLSD case. Significant transmission rate increases are also observed for all relevant distances. Namely, at $L=350 \mathrm{~km}$, a $125 \%$ increase is achieved with respect to the MF+symbol-by-symbol detection (see Fig. 4). Without any restrictions on power and state size the transmission rate obtained us-

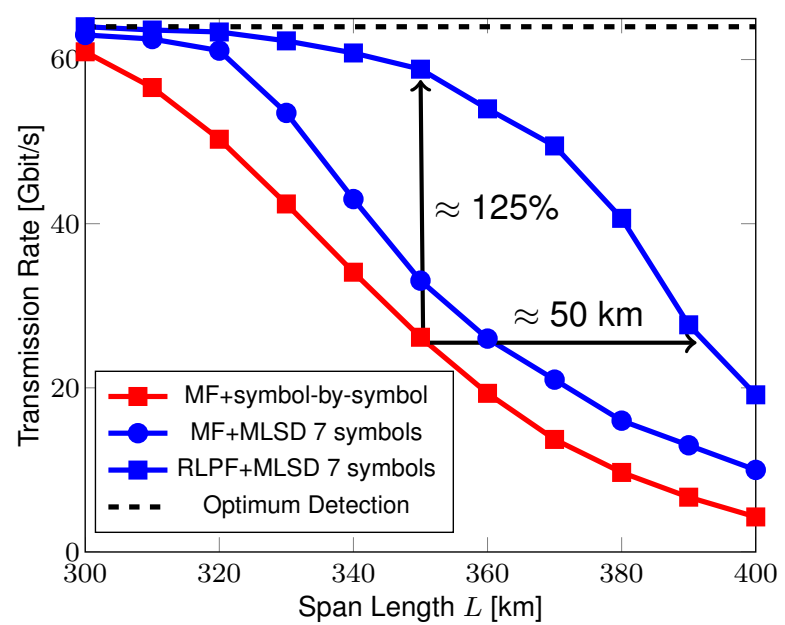

Fig. 4: Achievable transmission rates vs. $L$ for different detection strategies.

ing the RLPF+MLSD detector is expected to be constant across all distances (shown with a black dashed line in Fig. 4).

\section{Conclusions}

A near-optimum detection scheme, tailored to the nonlinear single span optical fibre system was described. Such a detector arbitrarily approaches the optimal one with the appropriate choice of the low-pass filter bandwidth, the sampling rate and number of states. Compared to the proposed scheme other commonly used detection strategies were shown to be strongly suboptimal and unable to mitigate nonlinear effects. Our results suggest instead that, regardless of the amount of optical nonlinearity, optimal detection in a single span optical fibre system achieves arbitrarily low $\mathrm{BER}$ as in the AWGN channel.

\section{Acknowledgements}

Financial support from the UK EPSRC Programme Grant UNLOC EP/J017582/1 and Huawei Technologies is gratefully acknowledged.

\section{References}

[1] M. Sorokina et al., "Regeneration limit of classical Shannon capacity", Nature Commun., 5(3861) (2014).

[2] D. Marsella et al., "Maximum Likelihood Sequence Detection for Mitigating Nonlinear Effects", J. Lightwave Technol., 32(5) (2014).

[3] N. V. Irukulapati et al., "Stochastic Digital Backpropagation", IEEE T. Commun., 62(11) (2014).

[4] A. Bononi et al. "Nonlinear signal-noise interactions in dispersion-managed links with various modulation formats", Opt. Fiber Technol., 16(2) (2010).

[5] T. M. Cover and J. A. Thomas, "Elements of Information Theory", Chap. 2, Wiley and Sons (2006).

[6] H. Meyr et al., "On Sampling Rate, Analog Prefiltering, and Sufficient Statistics for Digital Receivers", IEEE T. Commun., 42(12) (1994). 